\title{
Why Are You Smiling? In a Strategic Context, People's Affective Responses Reflect the Meaning of Android's Facial Expressions
}

\author{
Galit Hofree*, Paul Ruvolo*, Christopher Reinert, Marian S. Bartlett and Piotr Winkielman
}

\begin{abstract}
Facial expressions play an important role in human emotional communication. Observers' reactions to facial expressions can be simple (e.g., smiling to a smile). However, they can also reflect the contextual meaning of an expression (e.g., smiling to an opponent's frown). Our current study provides evidence for contextual modulation of human responses to facial expressions of a hyper-realistic android. Such modulation occurred when human participants engaged in a strategic game with or against a facially expressive robot. These findings suggest a high level of sophistication in facial communication between humans and robots that can be utilized in future robot design to promote natural social rapport with their users.
\end{abstract}

Index Terms-Cognition, Human Robot Interaction, Psychology, Emotion Recognition.

\section{INTRODUCTION}

$\mathrm{P}$ roducing quality interactions between robots and humans is a key goal in robotic development. Natural interactions with humans will enable robots to work as service providers and care givers in a variety of environments, such as education [1], and care for the elderly [2]. However, social and affective communication proves to be a challenge for current robot models [3]. Some believe that unlocking social intelligence is key to future advances in AI [3].

One important means of natural communication is facial emotional expressions, such as smiling or frowning. Interestingly, humans, and some other primates, have been found to spontaneously mimic such facial expressions [4-6]. This automatic behavior appears to be important for promoting empathy, recognition of others' emotional states, and generally creating a bond between people [7, 8]. Our previous work has shown that people spontaneously mimic an anthropomorphic robot, providing evidence that such a natural means of communication can exist between humans and robots [9].

Of course, human emotional expressivity is not only

G. Hofree is with the Department of Psychology, University of California, San Diego, La Jolla, CA 92093 USA. (e-mail: ghofree@ucsd.edu).

P. Ruvolo was with the Institute for Neural Computation, University of California, San Diego, La Jolla, CA 92093 USA. He is now with the Department of Computer Science, Bryn Mawr College, Bryn Mawr, PA 19010 USA (e-mail: pruvolo@ucsd.edu). C. Reinert is with the Department of Psychology, University of California, San Diego, La Jolla, CA 92093 USA. (e-mail: cmreiner@ucsd.edu).

M.S. Bartlett is with the the Institute for Neural Computation, University of California, San Diego, La Jolla, CA 92093 USA. (e-mail: marni@salk.edu).

P. Winkielman is with the Department of Psychology, University of California, San Diego, La Jolla, CA 92093 USA. (phone: 858-822-0682; fax: 858-534-7190; e-mail:

pwinkielman@ucsd.edu).

* These authors contributed equally to this work. grounded in mimicry reactions to others in our environment. Our emotional reactions to others' expressions also depend on the current context. For example, when playing bridge, you may respond positively to your partner's smile, since this is a sign that you are both winning. Yet, you may respond with a frown when your opponent is smiling, since this smile signals a loss for you. There is some debate on how automatic mimicry processes may interact with other emotive processes. Some evidence suggests that mimicry, as an automatic process, can interfere if our goal is not to mimic [10], or at least slow down non-mimicking reactions [11]. However, certain studies suggest that response to even unconscious cues can be modulated by the current context [12].

The current research shows that robotic emotional expressivity can elicit human emotional reactions in more complex ways than simple mimicry. Participants were asked to play a game with or against an android. On half of the trials, the android displayed truthful emotional reactions to his own outcome from the current game using his faces. On the other half the outcome of the game was displayed in writing on the computer terminal. Using electromyography (EMG) to measure people's facial reactions, we found that people's expressions are solely a function of participants' own outcome from the game. That is, participants smile when they win and frown when they lose. Critically, this occurs regardless of whether the information about win or loss is communicated by the android's smile or by his frown. Furthermore, the android's expressions elicited greater facial reactions in participants, as compared to their reactions in response to the outcome being displayed on the computer screen.

These findings demonstrate that people's facial responses to androids are open to contextual influences, and do not simply

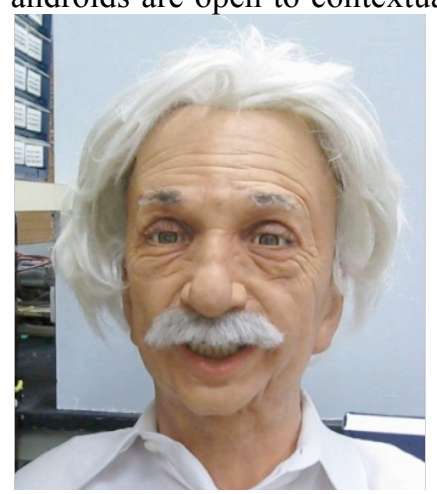
reflect mimicry. Overall, the results suggest that simple facial expressions can provide an important and subtle means of communicating emotional information and promoting emotional reactions beyond mimicry in human robot interactions.
Fig. 1. Einstein the robot, smiling. 


\section{METHODS}

\section{Participants}

36 University of California, San Diego undergraduates participated in the experiment (11 female). 9 subjects were excluded due to corrupt data.

\section{Robot Design}

This study utilized a state-of-the-art android manufactured by Hanson Robotics [13]. Its face, made of skin-like materials, is actuated by 31 servos (motors) programmed to perform facial movements that closely match human expressions, such as happiness and anger (see Fig. 1). The android's human-like appearance is enhanced by resemblance to Albert Einstein (henceforth, we refer to it as Einstein).

\section{Experiment Procedure}

Participants were first introduced to Einstein and asked to answer a few questions about him. The experiment itself consisted of repeated dice games, in which participants played to win either with or against Einstein. The games' outcome depended purely on chance. The final outcome was conveyed on each trial in one of two ways, as mentioned above. Participants' facial reactions were measured with facial electromyography - EMG. This technique, which measures electrical changes in underlying muscle activity, allows for fast and sensitive online assessment of participants' facial reactions to android's expressions. Measurements were taken from the cheek muscle, the zygomaticus (zygo.) major, used in smiling, and the brow muscle, the corrugator supercilii (corr.), used in frowning.

\section{RESULTS}

Overall, participants reliably responded facially to Einstein's facial expressions. Paired t-tests of mean zygo. and corr. activity during the $2^{\text {nd }}$ half of trials on which Einstein conveyed the outcome, comparing trials on which the participant won with those in which he lost, present evidence that participants significantly smile more during trials in which they win, than on trials which they lost (win: 0.318, lose 0.183 , stderr: $0.059 ; \mathrm{t}_{(26)}=2.301 ; \mathrm{p}=0.03$ ). Corr. activity presents a trend in the same direction (frowning more when losing than winning), although it is not significant (win: 0.165 , lose: -0.081 , stderr: $0.05 ; \mathrm{t}_{(26)}=-1.69, \mathrm{p}=0.10$ ).

Furthermore, participants' expressions reflected their outcome even when Einstein produced incongruent reactions, as was the case during the competitive block. We ran repeated measures MANOVAs across $500 \mathrm{~ms}$ intervals, comparing EMG activity during winning and losing trials (see Fig. 2). While zygo. activity showed a significant decrease between winning and losing trials during the cooperative block (outcome by time interaction: $\mathrm{F}_{(11,16)}=2.497, \mathrm{p}=0.047$ ), the corrugator showed a significant decrease between losing and winning trials during the competitive block (outcome by time interaction: $\left(\mathrm{F}_{(11,16)}=2.754, \mathrm{p}=0.032\right)$. These results suggest that participants did not simple mimic Einstein's expressions.

Finally, participants were more expressive when outcomes were conveyed by Einstein's expressions than through the computer, specifically during the competitive block. This added expressivity depended on participants' initial reactions to Einstein: those who described him using negative terms (such as "scary" or "creepy"), did not show this effect (3-way interaction: $\mathrm{F}_{(1,25)}=6.76, \mathrm{p}=0.015$; t-test comparing winning and losing reactions for those who did not react negatively, when Einstein conveyed the outcome: $\mathrm{t}_{(18)}=2.87, \mathrm{p}=0.011$ ).

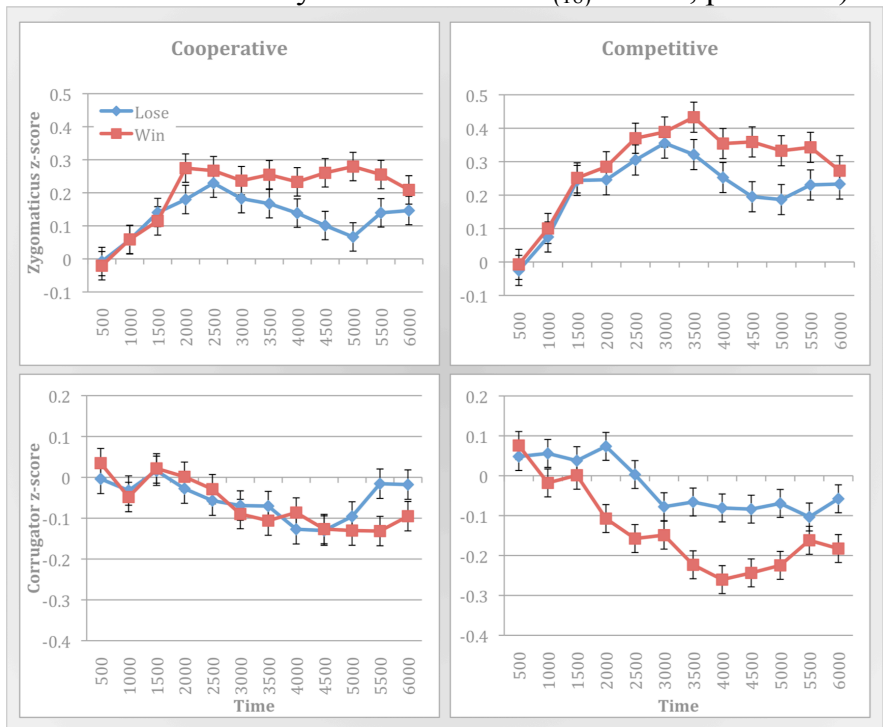

Fig. 2. Participants respond facially to Einstein's expressions in both the cooperative and competitive conditions. Zygo. (top) and Corr. (bottom) activity during trials in which Einstein conveys the outcome, compared across winning and losing trials, in either the cooperative (left) or the competitive (right) conditions.

\section{REFERENCES}

[1] A. N. Meltzoff, P. K. Kuhl, J. Movellan, and T. J. Sejnowski, "Foundations for a New Science of Learning," Science, vol. 325, no. 5938, pp. 284-288, Jul. 2009.

[2] S. Coradeschi, H. Ishiguro, M. Asada, S. C. Shapiro, M. Thielscher, C. Breazeal, M. J. Mataric, and H. Ishida, "Human-Inspired Robots," IEEE Intell. Syst., vol. 21, no. 4 pp. 74-85, Jul. 2006.

[3] K. Dautenhahn, "Socially intelligent robots: dimensions of human-robot interaction," Philosophical Transactions of the Royal Society B: Biological Sciences, vol. 362, no. 1480 , pp. $679-704$, Apr. 2007.

[4] U. Dimberg, "Facial reactions to facial expressions.," Psychophysiology, 1982

[5] D. N. McIntosh, A. Reichmann Decker, P. Winkielman, and J. L. Wilbarger, "When the social mirror breaks: deficits in automatic, but not voluntary, mimicry of emotional facial expressions in autism," Developmental Science, vol. 9, no. 3, pp. 295-302, 2006.

[6] M. Davila Ross, S. Menzler, and E. Zimmermann, "Rapid facial mimicry in orangutan play," Biology letters, vol. 4, no. 1, p. 27, 2008.

[7] T. L. Chartrand and J. A. Bargh, "The chameleon effect: the perception-behavior link and social interaction," J Pers Soc Psychol, vol. 76, no. 6, pp. 893-910, Jun. 1999.

L. Carr, M. Iacoboni, M.-C. Dubeau, J. C. Mazziotta, and G. L. Lenzi, "Neural mechanisms of empathy in humans: A relay from neural systems for imitation to limbic areas," Proceedings of the National Academy of Sciences, vol. 100, no. 9, pp. 5497-5502, Apr. 2003.

[9] G. Hofree, P. Ruvolo, M. S. Bartlett, and Pi. Winkielman, "Bridging the mechanical and human mind: Spontaneous facial mimicry of a hyper-realistic android," under review, 2012.

[10] R. Cook, G. Bird, G. Lünser, S. Huck, and C. Heyes, "Automatic Imitation in a Strategic Context: Players of Rock-Paper-Scissors Imitate Opponents' Gestures," Proc. R. Soc. B, vol. 279, no. 1729, pp. 780-786, Feb. 2012.

[11] M. Brass, H. Bekkering, and W. Prinz, "Movement observation affects movement execution in a simple response task," Acta Psychologica, vol. 106, no. 1-2, pp. 3-22, Jan. 2001.

[12] M. Tamir, M. D. Robinson, G. L. Clore, L. L. Martin, and D. J. Whitaker, “Are We Puppets on a String? The Contextual Meaning of Unconscious Expressive Cues," Pers Soc Psychol Bull, vol. 30, no. 2, pp. 237-249, Feb. 2004.

[13] T. Wu, N. J. Butko, P. Ruvulo, M. S. Bartlett, and J. R. Movellan, "Learning to Make Facial Expressions," in Proceedings of the 2009 IEEE 8th International Conference on Development and Learning, 2009, pp. 1-6. 